\title{
Large-eddy simulations of bushfire plumes in the turbulent atmospheric boundary layer
}

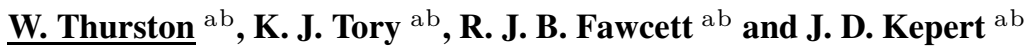 \\ ${ }^{a}$ The Centre for Australian Weather and Climate Research, Bureau of Meteorology, Docklands, Victoria \\ 3008, Australia \\ ${ }^{\mathrm{b}}$ Bushfire Cooperative Research Centre, Albert Street, East Melbourne, Victoria 3002, Australia \\ Email: w.thurston@bom.gov.au
}

\begin{abstract}
Spot fires are a hazardous phenomenon which can lead to unpredictable fire behaviour and accelerated fire spread. Spot fires occur when firebrands are lofted into strong ambient winds and ignite new fires downwind. Anecdotal evidence suggests that this lofting and transport of firebrands can be responsible for the ignition of spot fires at large distances, up to tens of kilometres ahead of the fire front. A thorough knowledge of the potential for lofting from a fire is therefore desirable in order to accurately predict the fires rate of spread and coverage.

The extent to which firebrands are lofted and transported away from a fire is largely determined by both the intensity of the fire convective column and the strength of the ambient winds. Previous work on the response of fire plumes to background winds has principally relied on theoretical or highly idealised numerical models. Here we use high-resolution three-dimensional numerical simulations, performed with the UK Met Office Large-Eddy Model, to investigate the behaviour of bushfire plumes. We begin by simulating the dry, neutral atmospheric boundary layer for a range of wind speeds. Simulations are run to a quasi-steady state, ensuring that the flow displays realistic turbulence properties. Plumes are then produced by imposing a localised positive surface heat flux anomaly at the model surface. The sensitivity of the size, shape and intensity of the plume's updraft to the interaction between the plume and the turbulent atmospheric boundary layer is explored, with reference to the potential for spotting.
\end{abstract}

Keywords: Bushfire plumes, large-eddy simulations 


\section{INTRODUCTION}

When firebrands are lofted to large heights above the fire from which they originate, they often travel faster and in a different direction to the near-surface winds, due to vertical wind shear. This means that fires spread by spotting can travel fast and are especially unpredictable. There is evidence of long-range spotting in excess of $30 \mathrm{~km}$ during the Black Saturday bushfires [e.g. Cruz et al., 2012]. It is therefore desirable to have a good understanding of the dynamics of bushfire plumes and updrafts, in order to assess the potential for spotting and the associated dangerous fire spread.

Previous observational work has suggested that interaction of the fire plume with the background wind is critical in determining the plume behaviour [e.g. Byram, 1959], essentially through a balance between the potential energy of the buoyant plume and the kinetic energy of the winds. Attempts to fully address this have been hampered by a lack of good quality observational datasets in consistent conditions that cover a wide enough parameter space of wind and fire intensities. Recently, computer power has increased to the point at which meteorological models are able to be run at high enough resolutions to explore the response of bushfire plumes to different background winds. However, often they have been used in a two-dimensional configuration or without spinning up a turbulent atmospheric boundary layer [e.g. Kiefer et al., 2009], which both impact the rate of entrainment of ambient air into the plume and therefore its dynamics.

In this study we initially use high-resolution, three-dimensional, large-eddy simulations to model the turbulent atmospheric boundary layer for a range of wind regimes. We then use the output of these realistic, turbulent simulations as initial conditions for idealised simulation of bushfire plumes under a range of wind regimes.

\section{THE NUMERICAL MODEL}

The simulations presented here are performed using Version 2.4 of the UK Met Office Large-Eddy Model (LEM) [Gray et al., 2001]. The LEM is a high-resolution non-hydrostatic atmospheric model which solves a Boussinesq equation set on a three-dimensional Cartesian grid. The sub-grid stresses are parametrized using a stability-dependent version of the Smagorinsky scheme, described by Brown et al. [1994].

Previously the LEM has been used for traditional high-resolution atmospheric applications, from dry boundary-layer turbulence to deep moist convection, although recently it has been used to study the behaviour of fire plumes. Devenish and Edwards [2009] used the LEM to perform simulations of the Buncefield oil depot fire in the UK, the largest fire in Europe since the Second World War. The model was able to successfully reproduce the observed height and multi-directional spread of the plume. Devenish et al. [2010] performed further validation by comparing the entrainment and radial spreading rates of buoyancy and vertical velocity in plumes simulated by the LEM to theoretical plume models.

\section{MODEL CONFIGURATION}

All simulations presented here are performed on a domain with horizontal dimensions of 768 by 384 grid points, with a uniform grid spacing of $50 \mathrm{~m}$ in both directions, resulting in a domain size of $38.4 \mathrm{~km}$ by $19.2 \mathrm{~km}$. The domain has 256 grid points in the vertical, made from a stretched grid specified with a vertical grid spacing of $10 \mathrm{~m}$ near the surface, which increases smoothly to $50 \mathrm{~m}$ above a height of $3 \mathrm{~km}$ and leads to a model top at $10 \mathrm{~km}$. The lateral boundary conditions of the model are doubly periodic and a no-slip condition is applied at the lower boundary, with a specified roughness length of $0.05 \mathrm{~m}$. The domain size chosen is large enough for the periodic lateral boundary conditions not to affect the simulated plumes. At the model's upper boundary, a free-slip condition is applied in conjunction with a Newtonian damping layer in the top $2 \mathrm{~km}$ of the domain, to prevent the reflection of vertically propagating gravity waves. Our simulations are performed without any radiation, moisture or microphysics and the Coriolis force is also ignored.

\section{BOUNDARY-LAYER SIMULATIONS}

As described in the Introduction, before we attempt to model the behaviour of fire plumes we first establish a set of realistic "hot-start" conditions, in which bushfire plumes can be subsequently simulated. The model is initialised with horizontally homogeneous conditions. An initial potential temperature profile is specified, consisting of a constant $300-\mathrm{K}$ mixed layer of $3 \mathrm{~km}$ depth, above which there is a constant lapse rate of $3 \mathrm{~K} \mathrm{~km}^{-1}$ up to the model top. This is designed to be representative of the vertical structure of the atmosphere on a typical Australian high fire risk day. The initial velocity is set to $u=u_{\text {top }}$ and $v=0$ throughout the domain. Small perturbations with a maximum amplitude of $\pm 0.1 \mathrm{~K}$ are added to the initial potential temperature field within the mixed layer, in order to initialise the development of turbulence, and then the 
W. Thurston et al., Large-eddy simulations of bushfire plumes...

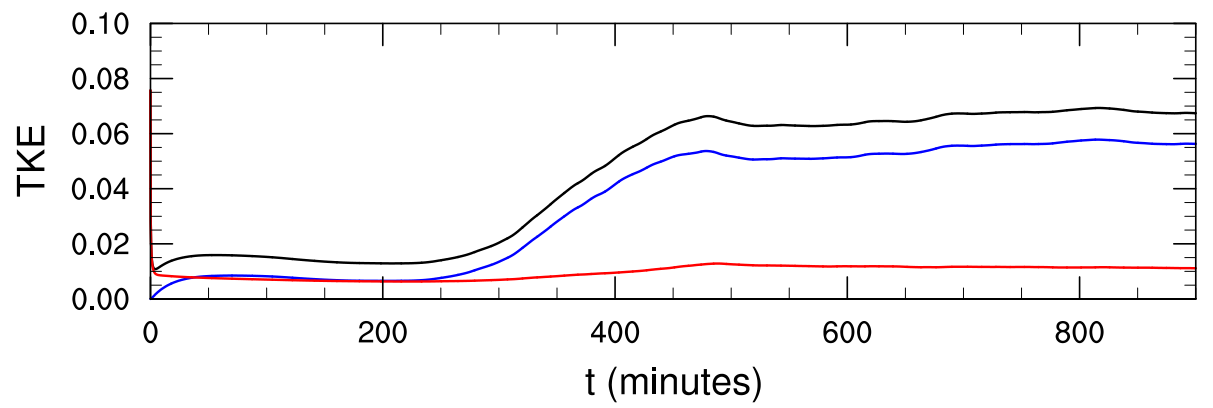

Figure 1. Timeseries of the domain-averaged turbulent kinetic energy (TKE) in the $10 \mathrm{~m} \mathrm{~s}^{-1}$ backgroundwind simulation. The resolved TKE is plotted in blue, the sub-grid TKE is plotted in red and the total TKE is plotted in black.
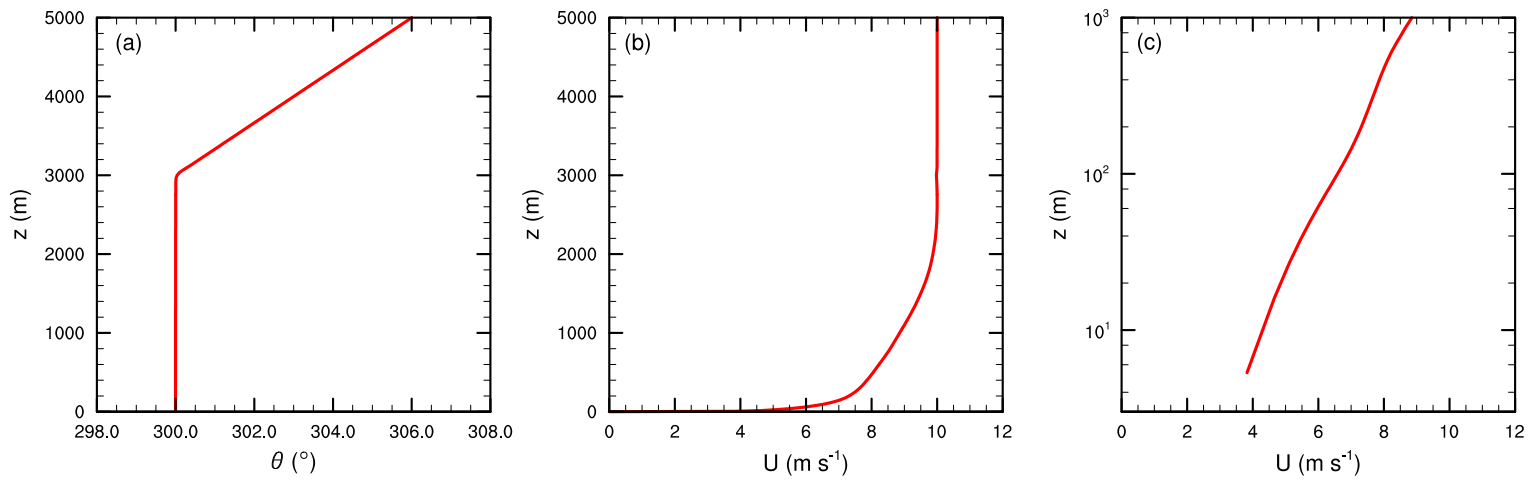

Figure 2. Domain-averaged profiles of the mean condition in the $10 \mathrm{~m} \mathrm{~s}^{-1}$ background-wind simulation at quasi-steady state. (a) Potential temperature, $\theta$; (b) velocity, $\mathrm{m} \mathrm{s}^{-1}$; and (c) velocity, $\mathrm{m} \mathrm{s}^{-1}$, plotted on a logarithmic height scale.

model is integrated forward in time with a variable timestep, determined by the Courant-Friedrichs-Lewy condition.

We perform six simulations in total, with six different values for the initial velocity, $u_{\mathrm{top}}$, of 2.0, 5.0, 7.5, 10.0, 12.5 and $15.0 \mathrm{~m} \mathrm{~s}^{-1}$. Figure 1 shows the timeseries of the domain-averaged turbulent kinetic energy (TKE) in the $10 \mathrm{~m} \mathrm{~s}^{-1}$ simulation. After about 550 minutes of model time the resolved mixed-layer turbulence has fully spun up and the model has reached a quasi-steady state. The form of the timeseries plots is similar for each value of $u_{\text {top }}$, although the simulations with higher values of $u_{\text {top }}$ reach quasi-steady state earlier in time than the simulations with lower values of $u_{\mathrm{top}}$. The exception to this is the $u_{\mathrm{top}}=2.0 \mathrm{~m} \mathrm{~s}^{-1}$ case, in which the resolved mixed-layer turbulence does not spin up at all.

The effect that spinning up the mixed-layer turbulence to a quasi-steady state has on the domain-averaged mean conditions is shown in Figure 2. The average potential temperature profile (Figure 2 (a)) has only been modified slightly from the specified initial conditions, with a small smoothing of the transition point at the top of the mixed layer where the constant potential temperature zone meets the constant potential-temperature gradient zone. The modification of the average velocity profile (Figure 2 (b)) is much more marked. The velocity at the surface has reduced to zero and a log layer has developed above it, as highlighted by the profile replotted on a log-height y-axis (Figure 2 (c)). In fact, the velocity at the surface reduces to zero very early on in the simulation, but the log layer only develops once the resolved mixed-layer turbulence has spun up (not shown). The domain-averaged profiles of the velocity variances, $\overline{u^{\prime} u^{\prime}}$ and $\overline{w^{\prime} w^{\prime}}$, and the vertical flux of horizontal momentum, $\overline{u^{\prime} w^{\prime}}$, are presented in Figure 3. The profiles of these quantities and their partitioning into resolved and sub-grid components are in good agreement with previous studies of the neutral, shear-driven boundary layer [e.g. Mason and Thomson, 1987; Andren et al., 1994; Moeng and Sullivan, 1994] indicating 
W. Thurston et al., Large-eddy simulations of bushfire plumes...
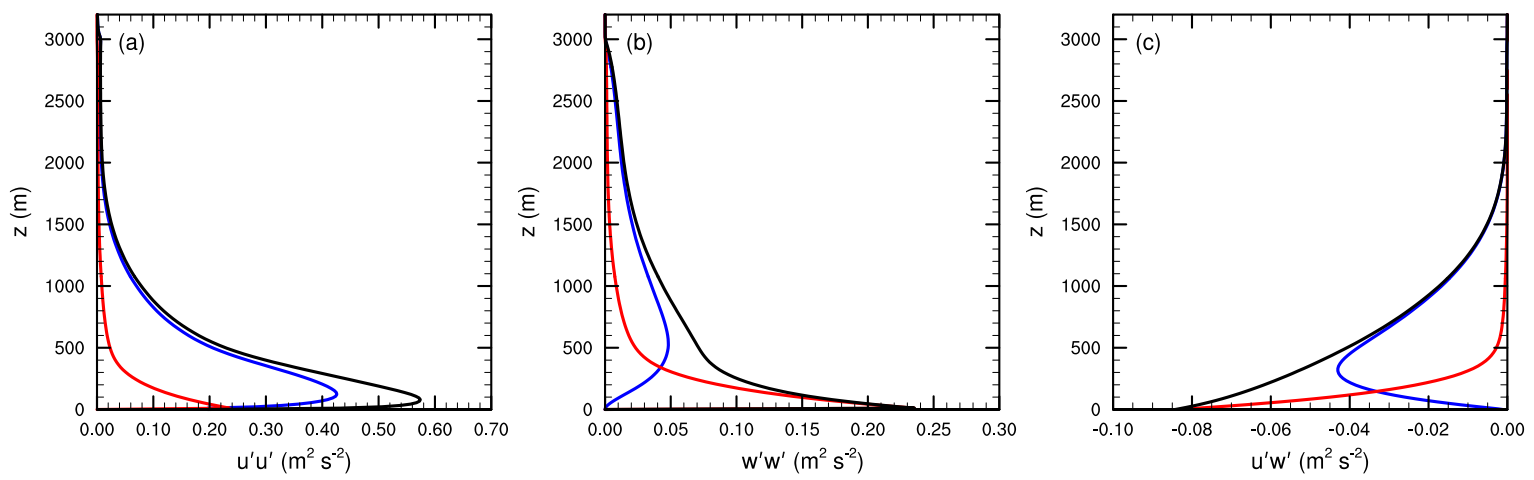

Figure 3. Domain-averaged profiles of the velocity variances and vertical momentum flux in the $10 \mathrm{~m} \mathrm{~s}^{-1}$ background-wind simulation at quasi-steady state. (a) $\overline{u^{\prime} u^{\prime}}, \mathrm{m}^{-2} \mathrm{~s}^{-2}$; (b) $\overline{w^{\prime} w^{\prime}}, \mathrm{m}^{-2} \mathrm{~s}^{-2}$; and (c) $\overline{u^{\prime} w^{\prime}}$, $\mathrm{m}^{-2} \mathrm{~s}^{-2}$. In each panel the resolved component is plotted in blue, the sub-grid component is plotted in red and the total is plotted in black.

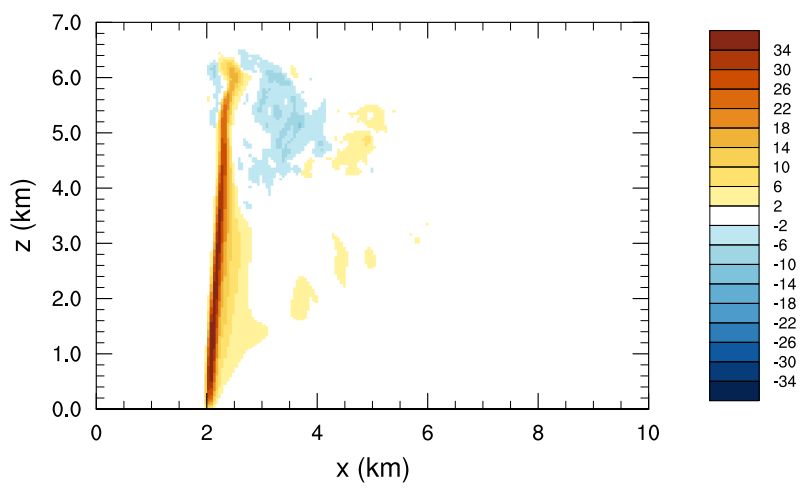

Figure 4. Snapshot of the instantaneous centreline vertical velocity, $\mathrm{m} \mathrm{s}^{-1}$, after 30 minutes of model time in the $2 \mathrm{~m} \mathrm{~s}^{-1}$ background-wind simulation.

that we have generated a realistic atmospheric boundary layer.

\section{PLUME SIMULATIONS}

We introduce an idealised bushfire plume into the simulations using a methodology similar to that of Devenish and Edwards [2009]. A circular heat flux anomaly, of radius $250 \mathrm{~m}$ and intensity $1 \times 10^{5} \mathrm{~W} \mathrm{~m}^{-2}$, is situated at the model's lower boundary, $2 \mathrm{~km}$ from the upwind boundary in the $x$-direction and equidistant from the lateral boundaries in the $y$-direction. It is to be noted that there is no direct feedback of the atmosphere back onto the fire in these simulations and therefore no capacity to investigate fire spread. Therefore, in this configuration it is possible to isolate and study just the interaction between the plume and background conditions, without the extra variable of the surface-fire behaviour.

The idealised surface "fire" is applied to all six background windspeed simulations and it is found that in each case, the resultant plume reaches a quasi-steady state after 30 minutes of integration time. The simulations are then integrated for a further 60 minutes to collect statistics of the "mean" plume behaviour, as well as instantaneous model output. Here, we first examine snapshots of the plume under the weakest $\left(2 \mathrm{~m} \mathrm{~s}^{-1}\right)$ and strongest $\left(15 \mathrm{~m} \mathrm{~s}^{-1}\right)$ background winds, to illustrate the plume behaviour under different regimes, before considering the "mean" plume behaviour for all wind conditions.

A snapshot of the instantaneous vertical velocity through the plume's centreline after 30 minutes of model time in the $2 \mathrm{~m} \mathrm{~s}^{-1}$ background-wind simulation is shown in Figure 4. The updraft is very narrow and also 


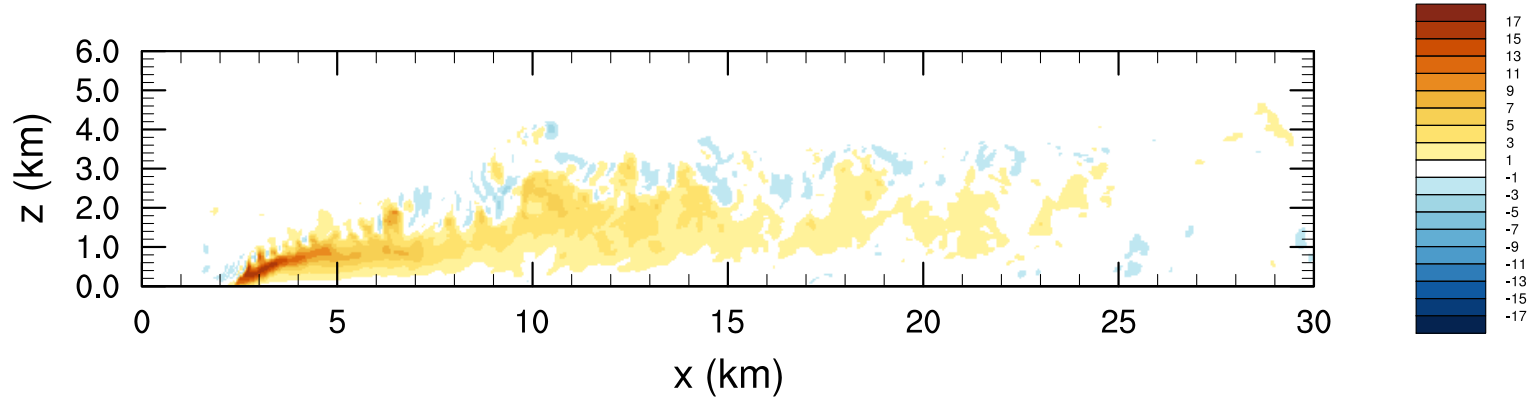

Figure 5. Snapshot of the instantaneous centreline vertical velocity, $\mathrm{m} \mathrm{s}^{-1}$, after 30 minutes of model time in the $15 \mathrm{~m} \mathrm{~s}^{-1}$ background-wind simulation.

very intense, with peak vertical velocities in excess of $40 \mathrm{~m} \mathrm{~s}^{-1}$. The plume is nearly vertically aligned and overshoots the top of the mixed layer by a height of more than $3 \mathrm{~km}$. Animation of the plume, not shown, reveals that the location of the plume is very steady, except in the region above the mixed layer, where there is some weak meandering as the plume decelerates. This behaviour is consistent with the weak turbulence of the background wind in this case.

The instantaneous vertical velocity snapshot of the plume with a $15 \mathrm{~m} \mathrm{~s}^{-1}$ background-wind (Figure 5) reveals substantially different behaviour of the plume under strong winds. In contrast to the $2 \mathrm{~m} \mathrm{~s}^{-1}$ background-wind case, the plume is very broad and bent over. It is much less intense, with a peak updraft of just over $16 \mathrm{~m} \mathrm{~s}^{-1}$, and does not extend beyond the top of the mixed layer. Animation of the plume, not shown, reveals that the plume is very turbulent, exhibiting strong meandering and pulsing, despite the lack of direct feedback of the atmosphere onto the fire.

The properties of the "mean" plume for each background windspeed, calculated from 60 minutes of model integration in each case, are shown in Figure 6. The maximum updraft strength (Figure 6 (a)) is found to vary smoothly from $41 \mathrm{~m} \mathrm{~s}^{-1}$ under a background windspeed of $2 \mathrm{~m} \mathrm{~s}^{-1}$ to $11 \mathrm{~m} \mathrm{~s}^{-1}$ under a background windspeed of $15 \mathrm{~m} \mathrm{~s}^{-1}$. The maximum updraft alone is not necessarily a useful predictor for firebrand transport, so we also plot contours of the $6 \mathrm{~m} \mathrm{~s}^{-1}$ updraft for each background windspeed (Figure 6 (b)). We chose this contour level as it is representative of a typical firebrand fall velocity [e.g. Ellis, 2010]. Here we can see that although under weak wind conditions the peak updrafts are of the order four times larger than under strong wind conditions, the area of each individual plume that contains an updraft strong enough to suspend a typical firebrand is actually quite similar.

\section{DISCUSSION AND CONCLUSIONS}

We have used the UK Met Office LEM to perform simulations investigating how idealised bushfire plumes vary in response to different background winds. We first took care to recreate realistic neutral, turbulent atmospheric boundary layers for a range of background windspeeds, under conditions designed to be representative of a typical high fire risk day. The plumes subsequently simulated across this range of windspeeds displayed a markedly different behaviour. Under weak background winds, plumes were intense, narrow, upright and smooth, whereas under strong background winds the plumes were comparatively weak, broad, bent over and turbulent. However, all plumes contained substantial areas of updrafts that would be sufficiently strong to suspend a typical firebrand. Although the idealised fire prescribed here was relatively intense and simulated under a wide range of wind conditions, the plumes simulated here would unlikely be strong enough to give rise to long-range spotting in excess of $30 \mathrm{~km}$. One potential reason for this is the lack of any moisture in either the fire or atmosphere, which is thought to play an important role in the dynamics of real-world fire plumes, particularly through the formation of pyrocumulonimbus [e.g. Cunningham and Reeder, 2009].

\section{ACKNOWLEDGMENT}

This research was partly supported by the Bushfire Cooperative Research Centre. We acknowledge the assistance of Adrian Hill and Ben Devenish (UK Met Office) in setting up the simulations. Thanks are also due to Andrew Sullivan (CSIRO) for useful discussions. 
W. Thurston et al., Large-eddy simulations of bushfire plumes...
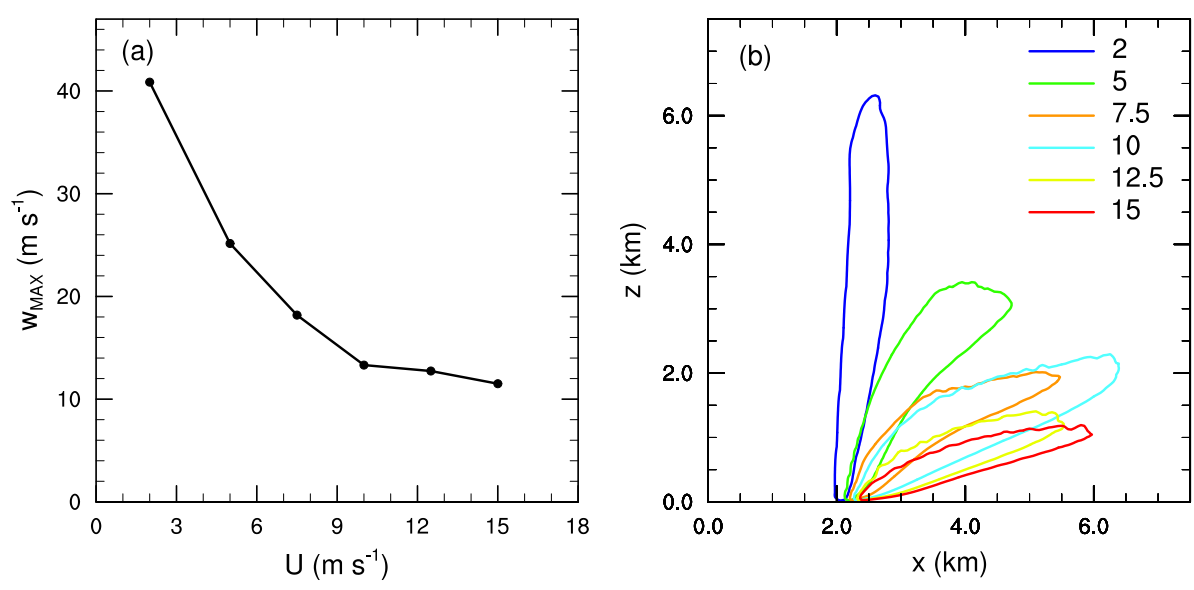

Figure 6. Properties of the plumes time averaged over one hour at quasi-steady state. (a) Maximum updraft speed, $\mathrm{m} \mathrm{s}^{-1}$, as a function of background windspeed, $\mathrm{m} \mathrm{s}^{-1}$; and (b) location of the $6 \mathrm{~m} \mathrm{~s}^{-1}$ updraft contour for each of the six background windspeed simulations.

\section{REFERENCES}

Andren, A., A. R. Brown, P. J. Mason, J. Graf, U. Schumann, C.-H. Moeng, and F. T. M. Nieuwstadt (1994). Large-eddy simulation of a neutrally stratified boundary layer: A comparison of four computer code. $Q$. J. Roy. Meteor. Soc. 120, 1457-1484.

Brown, A. R., S. H. Derbyshire, and P. J. Mason (1994). Large-eddy simulation of stable atmospheric boundary layers with a revised stochastic subgrid model. Q. J. Roy. Meteor. Soc. 120, 1485-1512.

Byram, G. M. (1959). Forest fire behavior. In K. P. Davis (Ed.), Forest Fire: Control and Use, pp. 90-123. New York: McGraw-Hill.

Cruz, M. G., A. L. Sullivan, J. S. Gould, N. C. Sims, A. J. Bannister, J. J. Hollis, and R. J. Hurley (2012). Anatomy of a catastrophic wildfire: The Black Saturday Kilmore East fire in Victoria, Australia. Forest Ecol. Manag. 284, 269-285.

Cunningham, P. and M. J. Reeder (2009). Severe convective storms initiated by intense wildfires: Numerical simulations of pyro-convection and pyro-tornadogenesis. Geophys. Res. Lett. 36.

Devenish, B. J. and J. M. Edwards (2009). Large-eddy simulation of the plume generated by the fire at the Buncefield oil depot in December 2005. Proc. R. Soc. A 465, 397-419.

Devenish, B. J., G. G. Rooney, and D. J. Thomson (2010). Large-eddy simulation of a buoyant plume in uniform and stably stratified environments. J. Fluid Mech. 652, 75-103.

Ellis, P. F. M. (2010). The effect of the aerodynamic behaviour of flakes of jarrah and karri bark on their potential as firebrands. J. Roy. Soc. West. Aust. 93, 21-27.

Gray, M. E. B., J. Petch, S. H. Derbyshire, A. R. Brown, A. P. Lock, H. A. Swann, and P. R. A. Brown (2001). Version 2.3 of the Met Office large eddy model: Part II. Scientific documentation. Turbulence and Diffusion Note 276, UK Met Office, Exeter, United Kingdom.

Kiefer, M. T., M. D. Parker, and J. J. Charney (2009). Regimes of dry convection above wildfires: Idealized numerical simulations and dimensional analysis. J. Atmos. Sci. 66, 806-836.

Mason, P. J. and D. J. Thomson (1987). Large-eddy simulations of the neutral-static-stability planetary boundary layer. Q. J. Roy. Meteor. Soc. 113, 413-443.

Moeng, C.-H. and P. P. Sullivan (1994). A comparison of shear- and buoyancy-driven planetary boundary layer flows. J. Atmos. Sci. 51, 999-1022. 